\title{
Two determinants of the peak shift in human voluntary stimulus generalization
}

\author{
ROBERT J. NEWLIN, JAMES P. RODGERS, and DAVID R. THOMAS \\ University of Colorado, Boulder, Colorado 80309
}

\begin{abstract}
Two hundred and forty college students were divided into two groups, with training stimuli (from the brightness dimension) selected to produce small and large adaptation level shifts between discrimination training (to respond "same" to $S+$ and "different" to $S-$ ) and generalization testing. These were further divided into three groups with discriminations expected to yield positive (toward brighter values), negative (toward dimmer values), or zero postdiscrimination peak shifts. Half the subjects received brief discrimination training while half received extended training. A further group of 60 subjects were given exposure to the stimuli comparable to that of the extended training subjects, but were asked to rate the stimuli instead of being given discrimination training. The results indicated that two independent, additive sources of shift were active. One source, occurring in all groups, was interpreted as being due to the change in adaptation level from training to test. The other source of shift, occurring only in the groups with extended discrimination training, was interpreted as due to the establishment of asymmetrical decision criteria; the more traditional interpretation in terms of the interaction between excitatory and inhibitory gradients of response strength was also considered.
\end{abstract}

One of the most robust findings in the study of discrimination learning is that of the peak shift in postdiscrimination stimulus generalization. Following acquisition of a successive discrimination between two stimulus values closely spaced on the same continuum, the peak (i.e., the point of maximal responding) is displaced from the $S+$ to a value farther removed from the $\mathrm{S}-$. This peak shift has been observed in several infrahuman species, as well as in children and adult humans, along several different stimulus dimensions, including wavelength, brightness, and line angle (cf. Purtle, 1973). The peak shift, at least in animals, has been most commonly interpreted in terms of Spence's (1937) theory, according to which response strength to any stimulus is a consequence of the summation of generalized excitatory strength from $\mathrm{S}+$ and inhibitory strength from $\mathrm{S}-$.

A more recent interpretation of generalization gradients involves the application of statistical decision theory (Boneau \& Cole, 1967). According to this approach, any given physical stimulus produces a subjective experience (within the organism) called a discriminal process. Because the discriminal process is variable, repeated presentations of the same physical stimulus generate a discriminal distribution. The subject must then establish a criterion (or criteria) which

This research was supported by NICHD Research Grant HD03486 and NSF Research Grant BNS78-01407. The authors would like to thank John C. Inmann for his assistance in conducting these experiments. Requests for reprints should be addressed to: Dr. David R. Thomas, Department of Psychology, University of Colorado, Boulder, Colorado 80309. defines a range of the discriminal distribution that the subject will attribute to a particular stimulus. Blough (1969) has shown that peaked generalization gradients would be found if instructions (for human subjects) or single-stimulus training (for animal subjects) caused them to create two symmetrical response criteria around the mean discriminal process produced by the training stimulus. Blough also noted that peak shift or asymmetrical gradients could be accounted for by assuming that the two criteria were independent. Specifically, a peak shift such as predicted by Spence's theory would result if discrimination training caused the criterion on the $\mathrm{S}-$ side of $\mathrm{S}+$ to be moved toward $\mathrm{S}+$ and had little effect on the opposite criterion. This would mean that stimuli just to the opposite side of $\mathrm{S}+$ from $\mathrm{S}$ - would be more likely to produce a discriminal process that would be interpreted as $S+$ than those on the $S-$ side or the $\mathrm{S}+$ value itself.

Historically, the alternative to "absolute" approaches such as Spence's has been the relational view of Krechevsky (1938). According to this view, the subject solves the discrimination problem relationally by learning to respond to the larger (or smaller), the brighter (or dimmer), etc., of the training stimuli. A problem for this simple relational view is that the postdiscrimination gradient shows a peak, i.e., a single maximal value, beyond which responding decreases as stimuli still farther from $\mathrm{S}+$ are tested.

A problem for all of these approaches is the fact that peak shifts have been found in studies of generalizations in humans in which single-stimulus train- 
ing, rather than discrimination training, has been administered. For example, Thomas and Jones (1962) gave their subjects a 60 -sec exposure to a $525-\mathrm{nm}$ training stimulus with instructions to remember that stimulus so that they could identify it during a subsequent wavelength test. The location of the training stimulus in the test series was systematically varied, with the training stimulus being in the center of the range of test stimuli for one group and displaced to different extents from the center for four other groups. Only with the symmetrical group did a symmetrical generalization gradient (with a peak at the training stimulus) result. For the other groups, the peak was shifted toward the center of the test series. Capehart, Tempone, and Hébert (1969) proposed that this "central tendency effect"' was explicable in terms of Helson's $(1947,1964)$ adaptation level (AL) theory. According to this theory, subjects perceive and remember stimuli not as absolute values but in relation to some internal representation of the "average" of previous experience with that dimension, called adaptation level. ${ }^{1}$ Presumably, in the Thomas and Jones study the training stimulus was established as the initial AL. As AL changed during testing, moving toward the central value of the (asymmetrical) test series, subjects continued to respond to that stimulus which was closest to AL at any given time. This interpretation is strongly supported by a study by Newlin, Rodgers, Dickson, Strub, and Thomas (1978) using the brightness dimension. These investigators demonstrated over a wide range of training and test conditions that the amount of shift in the peak of generalization gradients correlated highly with changes in $\mathrm{AL}$ as measured in independent groups. These groups viewed the same stimuli as did generalization test groups but gave ratings so that $\mathrm{AL}$ could be calculated as that stimulus given an average rating (4 on a scale of 1 to 7: cf. Helson, 1964; Parducci, 1963).

In order to account for the occurrence of a peak shift after discrimination training, Thomas, Svinicki, and Vogt (1973) extended a proposal by Capehart et al. (1969) and James (1953). Thomas et al. (1973) state their hypothesis as follows: "Suppose that a subject is trained to respond to a $200 \mathrm{gm}$. weight $(\mathrm{S}+)$ and not to respond to a $100 \mathrm{gm}$. weight $(\mathrm{S}-)$, and subsequently he is tested for generalization to a wide range of weight values. Presumably, equal experience with the two training stimuli will establish an $\mathrm{AL}$ somewhere between them, e.g., at the midway point $(150 \mathrm{gm})$. The $S$ may then learn to respond to a value of $50 \mathrm{gm}$. greater than AL. During generalization testing in which the $200 \mathrm{gm} \mathrm{S}+$ is the central stimulus, as is typical practice in studies of this sort, AL should drift toward the $200 \mathrm{gm}$. value. If $S$ continues to respond to a value $50 \mathrm{gm}$. heavier than $\mathrm{AL}$, maxi- mal responding should gradually shift from the previous $\mathrm{S}+$ toward a value of $50 \mathrm{gm}$. heavier. This explains the post-discrimination "peak shift" in $\mathrm{AL}$ terms, and it leads to an intriguing additional prediction. Suppose that in original training the $S+$ had been $200 \mathrm{gm}$., the $\mathrm{S}-, 180 \mathrm{gm}$., and the AL had been half-way between at $190 \mathrm{gm}$. If, in testing $200 \mathrm{gm}$. were again the central value, a shift in peak responding of only $10 \mathrm{gm}$. would be expected. Thus, this application of adaptation-level principles leads to the prediction that the greater the $\mathrm{S}+\mathrm{S}-$ separation, the greater the resulting peak shift"' (p. 211). The AL view of Thomas et al. (1973) involves two different relations. First, it is assumed that $S+$ and $S-$ are encoded relative to an $\mathrm{AL}$ value $(\mathrm{S}=\mathrm{AL} \pm \times)$. Second, the peak shift can only be understood in relation to the $\mathrm{AL}$ of the generalization test series. This latter point is unique to the $A L$ position, since neither the absolute view of Spence (1937) nor the relational view of Krechevsky (1938) takes into account the composition of the generalization test series.

Thomas et al. (1973) tested their hypothesis in an experiment with adult human subjects, the brightness continuum, and three different magnitudes of S $+\mathrm{S} 1$ difference. In half of the groups, the $\mathbf{S}+$ was the brighter value and in half it was the dimmer. The results of the Thomas et al. study were generally in accord with their prediciton. The amount of peak shift was positively related to $\mathrm{S}+\mathrm{S}$ - difference, although the group differences were statistically significant only for the three cases in which the $\mathrm{S}+$ was a brighter value than the $\mathrm{S}-$. It should be noted that the results of two operant studies with pigeons (Hanson, 1959; Thomas, 1962) had shown a negative relationship between $\mathrm{S}+\mathrm{S}$ - separation and amount of peak shift, as predicted by Spence's theory (1937) and also by decision theory, since the closer stimuli would require more displacement of the decision criterion in order to perform the discrimination accurately.

More recently, Galizio and Baron (1979) trained human subjects to respond to two positive stimuli (two tones varying in pitch) but not to respond to a third stimulus (S-) midway between the other two. A generalization test centered on the $S-$ stimulus produced a gradient with a minimum at $\mathrm{S}-$ and increased responding in both directions as stimuli became more different from $\mathrm{S}-$. Since the mean (and midpoint and median) of the training and test series were the same, no AL shift would be expected. Furthermore, an AL shift could not account for the bidirectional peak shift obtained. The authors interpreted their results in terms of Spence's theory, but, as noted, a decision theory explanation would yield the same expectations. The finding by Galizio and Baron 
(1979) has been replicated in our laboratory (White \& Thomas, Note 1) so there is no question of its reliability. Unfortunately, Galizio and Baron made no attempt to reconcile the discrepancy between their findings (and those in the animal literature), which are inconsistent with the AL view, and those of the various other studies referred to above, which are consistent with only the $A L$ interpretation.

This review of the relevant literature suggests that there may be two different sources of peak shift, one unique to the discrimination learning paradigm, possibly reflecting processes such as those postulated by Spence, and one which is more ubiquitous, resulting from $A L$ shifts between training and testing whether they occur in single-stimulus or discrimination training paradigms. There is reason to believe that the type of result obtained may depend on the amount of training administered. As Thomas (1974) pointed out, in operant studies with pigeons, substantial overtraining is given so that generalization testing can be carried out in extinction. Thomas (1962) demonstrated that the size of the postdiscrimination peak shift is positively related to the amount of training for pigeons. On the other hand, extended training might reduce the amount of $\mathrm{AL}$ shift obtained; extended training could "anchor" the AL such that stimuli during the generalization test have relatively less ability to modify AL. Giurintano (reported in Thomas \& Fenner, 1978), using single-stimulus training with the line angle dimension, followed by asymmetrical testing, found that the more training administered, the less the amount of shift obtained. This may explain why the animal studies have produced only results consistent with Spence's view; in these studies, there could be little or no AL shift from training to testing. In human studies, instructions make extended training unnecessary, and with brief training it may be that the excitatory and inhibitory processes postulated by Spence to underlie intradimensional learning do not have a chance to develop, or develop only very weakly. Where large AL shifts also occur, it may be difficult to demonstrate Spence-type effects. Where no AL shift takes place, as in the Galizio and Baron study, Spence-type effects may then be revealed.

Hébert (1973) has suggested a model of human stimulus generalization after single-stimulus training which combines decision theory and AL theory. According to this model, decision theory predicts the slope of the gradient but not the location of the peak, whereas AL theory predicts the location of the peak but not the slope of the gradient. The results of Thomas et al. (1973) conform to this model if we assume that the shift due to AL change from training to test was large in relation to any asymmetry of decision criteria. The results of Hanson (1959) and
Thomas (1962) would also be predicted if one assumed that the increased training used in these studies, in lieu of the instructions usually given to human subjects, reduced the amount of $A L$ shift between training and test until it was small and added the assumption of the asymmetry of the decision criteria as a further determinant of the peak of the gradient. In the Galizio and Baron (1979) study there should be no shift in AL to interfere with detecting a peak shift due to other processes.

This analysis would gain credence if it could be shown in a single experimental paradigm that evidence for both Spence-type and AL effects could be obtained depending on the amount of training administered. Experiment 1, with minimal discrimination training, and Experiment 2, with maximal training, accomplished this objective. Experiment 3 determined that the Spence-type results obtained in Experiment 2 required that discrimination training (i.e., practice responding "same" to one stimulus and "different" to another with feedback on correctness) be employed rather than mere exposure to the same training stimuli without feedback.

\section{EXPERIMENT 1}

In Experiment 1, minimal training was given with the expectation that the results thus obtained would support predictions based on the AL theory. Groups and conditions were established so that in many cases peak shifts predicted on the basis of the AL and excitation-inhibition views differed in their relative magnitude and even in their direction from the $\mathrm{S}+$ training stimulus.

Nine (logarithmically) equally spaced brightness values were used in testing, and the test $\mathrm{AL}$, determined empirically in a category rating task with a separate group of subjects, was slightly less than a stimulus value (SV) of 6 (i.e., fourth brightest). ${ }^{2}$ Three experimental groups received successive discrimination training designed to produce a training AL near (but actually slightly above) SV 3. One (control) group learned to respond to SV $3(\mathrm{~S}+)$ with two symmetrical $S-s$, one at SV 1 and the other at SV 5. According to AL theory, this group should show a peak shift from SV 3 toward SV 6. A second group learned to respond to SV $4(S+)$ but not to SV 2 (S-). This group should show a peak shift to a higher value than the control, since this group presumably learned to respond to a value greater than their training AL. A third group received the opposite training, that is, to respond to SV $2(S+)$ and not to SV $4(\mathrm{~S}-)$. For analogous reasons, this group should show a peak shift to a lower value than the control. According to AL theory, all groups should shift in a positive direction, i.e., toward brighter 
stimuli as the AL shifts from its training value (slightly greater than SV 3 ) to its ultimate test value (slightly less than SV 6). The three gradients should shift by an equal amount when computed with respect to the original $S+$ value, since the three $\mathrm{AL}$ values presumably shift by an equal amount. In terms of Spence's theory, however, the group with SV 2 as S + should shift in a negative direction, i.e., toward dimmer values, and the group with SV 3 as $S+$ and two symmetrical $S-s$ should not shift at all.

Three more experimental groups received similar successive discrimination training designed to produce a training AL slightly, above SV 6 . The control group learned to respond to $S+$ at SV 6 with $S-s$ at SV 4 and SV 8, a second group learned to respond to SV 7 $(\mathrm{S}+)$ but not to SV $5(\mathrm{~S}-)$, and a third group learned to respond to SV $5(\mathrm{~S}+)$ but not to SV $7(\mathrm{~S}-)$. For these groups, the shifts predicted by Spence's theory are the same as those for the comparable groups described earlier. According to $\mathrm{AL}$ theory, however, all shifts should be substantially reduced in magnitude, and they should be in the negative direction as the training AL (slightly above SV 6) is replaced by the test AL (slightly below SV 6).

\section{Method}

Subjects. The subjects were 60 male and 60 female students enrolled in introductory psychology courses at the University of Colorado.

Apparatus. Each subject was seated $60 \mathrm{~cm}$ in front of a 60 $\mathrm{cm}$-square panel which was covered with black felt cloth. At approximately eye level, a $2.7-\mathrm{cm}$-diam aperture was present. The subjects viewed a disk of white light projected onto a translucen glass screen behind the aperture. The light source was a light discrimination apparatus manufactured by the Lafayette Instrument Company (Model No. 14011), using a 60-W Sylvania clear Decor Lite (60CA9C/BL). Nine different light intensities were selected so as to be $.188 \log$ units apart. The intensity values and their experimental designations were: stimulus value (SV) 1 , $1.97 \mathrm{fL}$; SV 2, 3.04 fL; SV 3, $4.69 \mathrm{fL}$; SV 4, 7.23 fL; SV 5, $11.2 \mathrm{fL}$; SV 6, $17.2 \mathrm{fL}$; SV 7, 26.5 fL; SV 8, $40.9 \mathrm{fL}$; and SV 9, $63.0 \mathrm{fL}$. The experiment was conducted in a small, dimly illuminated room. The light reflected from the disk when it was not illuminated was approximately $.01 \mathrm{fL}$.

Procedure. The subjects were unsystematically assigned to two major groups (training $A L=S V 3$ or training $A L=S V 6$ ) and to one of three subgroups ( $\mathrm{S}+$ low, $\mathrm{S}+$ centered, $\mathrm{S}+$ high). Each of these six groups contained 10 male and 10 female subjects.

After the subject was seated in front of the stimulus panel, the following instructions were read: "This is an experiment in brightness perception. A light will be presented repeatedly through a small hole in the screen in front of you. Each time it will be presented for five seconds and may have a different brightness. The first brightness is called the test brightness. Try to remember this brightness because you will have to distinguish it from all the other brightnesses. When you do recognize the test brightness say 'same'. If a subsequent brightness is different from the test brightness say 'different.' Remember, each time the light is presented it will stay on for only five seconds, so try to respond while the light is on. I will tell you whether you are correct on the first few trials; then you will continue without further help. The first light is the test brightness. Keep its brightness in mind. For every light after that, say only 'same' or 'different'. Any questions?"
Only questions dealing with the procedure were answered by the experimenter. Following any needed clarifications of instructions, each subject was shown the appropriate $\mathbf{S}+$ stimulus. Each subject in the single $S-$ conditions was then shown five series of the two stimuli (10 stimulus presentations) and each subject in the double $\mathrm{S}$ - conditions was shown four series of three stimuli (12 stimulus presentations). After each correct response, the experimenter said "correct," and after each incorrect response, the experimenter said either "no, that was different from the original," or "no, that was the same as the original," depending on the error. Next, with no interruption in the procedure, all subjects were shown six series of all nine stimuli; feedback was no longer given to the subject. The sitmuli were randomized within each series in both discrimination training and generalization testing, and the interstimulus interval ranged unsystematically from $3-6 \mathrm{sec}$. Table 1 shows the amount of discrimination training and the discriminative stimuli for the six groups of Experiment 1 in Columns 1 and 2, respectively.

\section{Results and Discussion}

A gradient of stimulus generalization was constructed for each subject consisting of the number of "same" responses made to each of the test stimuli. The mean of these gradients (mean stimulus to which the subject said "same") was used as a measure of central tendency reflecting the locus of maximal responding (cf. Thomas \& Jones, 1962). To make this measure comparable for the three subgroups within each major discrimination training group $(\mathrm{AL}=$ SV 3 and $A L=S V$ ), a comparable portion of the entire generalization gradient was used. For the SV 3 groups, this consisted of the $\mathrm{S}+$ value, one stimulus value below it, and five stimulus values above it. For the SV6 groups, the values used were the $S+$, four stimuli below $S+$, and two stimuli above it. The means of these individual subject means for each group are presented in Column 3 of Table 1. Column 4 of Table 1, titled "Group Mean Shift," shows the total shift of the group and is, simply, the difference between the group's gradient mean (Column 3) and the group's S + (Column 2). The quantity represents the empirically determined shift of the stimulus called "same" between the end of discrimination training and generalization testing.

Figure 1 shows the generalization gradients, relative to $\mathrm{S}+$, of the three groups with training $\mathrm{AL}=$ SV 3 along with the mean of each gradient. All three groups shifted approximately two SV units toward brighter values. The highly comparable performance of these three groups is entirely in accord with AL theory. Recall that, from Spence's theory, no peak shift at all was predicted in the group with $S+=$ SV 3, and the shift obtained in the $S+=$ SV 2 group was in the direction opposite that predicted. Indeed, the gradient of this latter group peaked at its $S$ - value!

Figure 2 shows the generalization gradients for the three groups with training $A L=S V 6$. Recall that AL theory predicted a slight shift in the negative direction and that was what was found. The means of the three gradients are all slightly below the S+ value. $^{3}$ 
Table 1

Design of Experiments, Group Gradient Means, and Different Sources of Peak Shift

\begin{tabular}{|c|c|c|c|c|c|c|c|c|}
\hline \multirow[b]{2}{*}{1} & \multicolumn{3}{|c|}{2} & \multirow[b]{2}{*}{3} & \multirow[b]{2}{*}{4} & \multirow[b]{2}{*}{5} & \multirow[b]{2}{*}{6} & \multirow[b]{2}{*}{7} \\
\hline & - & + & - & & & & & \\
\hline \multicolumn{9}{|c|}{ Experiment 1} \\
\hline $\begin{array}{l}10 \\
12 \\
10\end{array}$ & $\begin{array}{l}1 \\
2\end{array}$ & $\begin{array}{l}2 \\
3 \\
4\end{array}$ & $\begin{array}{l}4 \\
5\end{array}$ & $\begin{array}{l}3.528 \\
4.806 \\
5.853\end{array}$ & $\begin{array}{l}1.518 \\
1.806 \\
1.853\end{array}$ & 1.729 & $\begin{array}{r}-.201 \\
.077 \\
.124\end{array}$ & .325 \\
\hline $\begin{array}{l}10 \\
12 \\
10\end{array}$ & $\begin{array}{l}4 \\
5\end{array}$ & $\begin{array}{l}5 \\
6 \\
7\end{array}$ & $\begin{array}{l}7 \\
8\end{array}$ & $\begin{array}{l}4.863 \\
5.677 \\
6.511\end{array}$ & $\begin{array}{l}-.137 \\
-.323 \\
-.489\end{array}$ & -.316 & $\begin{array}{r}.179 \\
-.007 \\
-.173\end{array}$ & -.352 \\
\hline \multicolumn{9}{|c|}{ Experiment 2} \\
\hline $\begin{array}{l}40 \\
42 \\
40\end{array}$ & $\begin{array}{l}1 \\
2\end{array}$ & $\begin{array}{l}2 \\
3 \\
4\end{array}$ & $\begin{array}{l}4 \\
5\end{array}$ & $\begin{array}{l}2.803 \\
4.242 \\
5.415\end{array}$ & $\begin{array}{r}.803 \\
1.242 \\
1.415\end{array}$ & 1.153 & $\begin{array}{r}-.350 \\
-.089 \\
.262\end{array}$ & .612 \\
\hline $\begin{array}{l}40 \\
42 \\
40\end{array}$ & $\begin{array}{l}4 \\
5\end{array}$ & $\begin{array}{l}5 \\
6 \\
7\end{array}$ & $\begin{array}{l}7 \\
8\end{array}$ & $\begin{array}{l}4.227 \\
5.414 \\
6.923\end{array}$ & $\begin{array}{l}-.773 \\
-.586 \\
-.077\end{array}$ & -.479 & $\begin{array}{r}-.294 \\
-.107 \\
.402\end{array}$ & .696 \\
\hline \multicolumn{9}{|c|}{ Experiment 3} \\
\hline $\begin{array}{l}40 \\
42 \\
40\end{array}$ & $\begin{array}{l}4 \\
5\end{array}$ & $\begin{array}{l}5 \\
6 \\
7\end{array}$ & $\begin{array}{l}7 \\
8\end{array}$ & $\begin{array}{l}4.987 \\
5.131 \\
6.417\end{array}$ & $\begin{array}{l}-.013 \\
-.869 \\
-.583\end{array}$ & -.488 & $\begin{array}{r}.475 \\
-.381 \\
.095\end{array}$ & -.570 \\
\hline
\end{tabular}

Note-Column 1 = amount of discrimination training. Column 2 = discriminative stimuli. Column $3=$ mean of gradient means. Column $4=$ group mean shift. Column $5=$ mean shift for triad. Column $6=$ deviation of group mean from triad mean. Column 7 = differential shift (Group 3 - Group 1). See text for definitions and further explanation.

In the centered S + groups, Spence's theory predicts no peak shift; however, the obtained shift can be attributed to a shift in AL from training to test values. The amount of shift in these centered groups is thus an estimate of that AL shift. Presumably, a comparable AL shift also occurs in the high and low $\mathrm{S}+$ groups; however, in these groups, it is possible to argue that in addition to this AL shift there is a peak shift which results from the interaction of excitatory and inhibitory processes as postulated by Spence or a shift in decision criteria. Since, in these two groups, gradient interaction shifts are presumably of equal magnitude and opposite in direction, they should, when added together, cancel out, so that the average of the three peak shift scores constitutes the best estimate of the shift due to AL factors alone. This number is shown in Column 5 of Table 1 and is labeled "Mean Shift for Triad." As can be seen, this shift is large and positive, 1.729, for the groups with training $A L=S V 3$, and much smaller and negative, -.316 , for the groups with training $\mathrm{AL}=$ SV 6, as was predicted by AL theory. Both of these shifts are significantly different from zero $[\mathrm{t}(285)=$ 15.71 and 2.87 , respectively, ps $<.01] .{ }^{4}$ Column 6 shows the deviation of groups' shifts from the triad's shift; note that the groups with symmetrical $S-s$ have very small deviations.

As mentioned above, even though most of the shift seems to be attributable to changes in $\mathrm{AL}$ between training and test, it is possible that there is also a shift attributable to Spence-type processes. The difference in the amount of peak shift between the high and low $S+$ groups (see Column 6) within each major discrimination group $(\mathrm{AL}=\mathrm{SV} 3$ and $\mathrm{AL}$ = SV 6) provides a measure of peak shift attributable to these processes. This measure, called "Differential Shift," is presented for both major groups in Table 1, Column 7. In fact, the group with SV 4 as S+ shows a greater shift from its $\mathbf{S}+$ than the group with $\mathrm{S}+$ $=$ SV 2 (difference $=.325$ ) in a direction that is consistent with Spence's theory. However, the difference in the groups with $\mathrm{AL}=\mathrm{SV} 6(-.352)$ is of equal magnitude and in a direction opposite to that predicted by Spence. Neither of these differential shifts is large enough to reach significance $[F(1,285)$ $=1.45$ and 1.70 , respectively, $\mathrm{ps}>.05$ ].

\section{EXPERIMENT 2}

In Experiment 2, we reexamined the six conditions used in Experiment 1 but increased the number of training trials by a factor of four. It was our expectation that this change would produce results consistent with Spence's theory. There were two possible reasons for this. Overtraining might anchor AL such that changes in AL from training to test would be

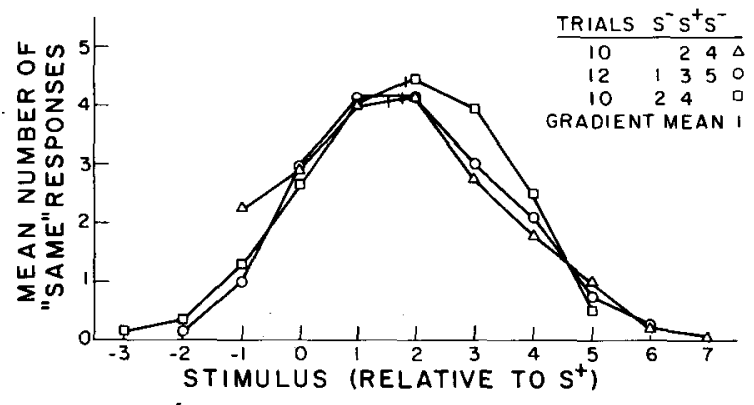

Figure 1. Generalization gradients and gradient means (mean of individual subject's gradient means; see text for further explanation) for groups with brief discrimination training and training ALs of SV 3. S + is labeled zero on the abscissa.

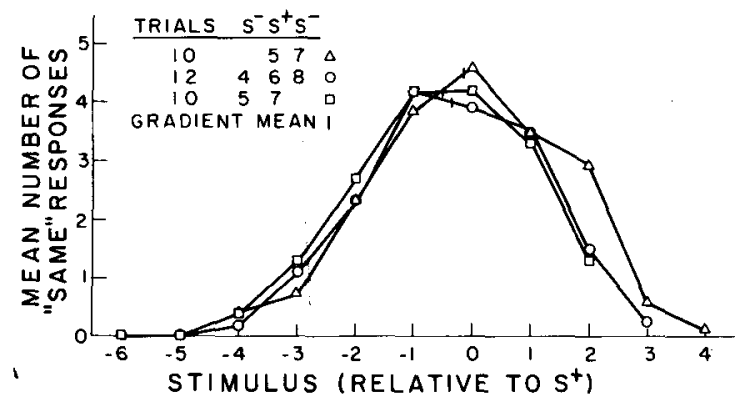

Figure 2. Generalization gradients and gradient means (mean of individual subjects' gradient means; see text for further explanation) for groups with brief discrimination training and training ALs of SV 6. $\mathbf{S}+$ is labeled zero on the abscissa. 
minimized, reducing or minimizing this source of shift. Furthermore, and more directly, increased training might permit the buildup of excitatory and inhibitory tendencies to the extent that these effects would be discernible.

\section{Method}

Subjects. The subjects were 60 male and 60 female students enrolled in introductory psychology courses at the University of Colorado.

Apparatus. The apparatus was the same as that used in Experiment 1.

Procedure. The procedure was identical to that of Experiment 1, except that each subject with a single $S$ - received 20 series of two stimuli (40 stimulus presentations) and each subject with two $S-s$ received 14 series of three stimuli (42 stimulus presentations) during training.

\section{Results and Discussion}

As can be seen in Table 1, the gradient means of all groups again shifted in the direction predicted by AL theory. The mean shift in the groups with training $\mathrm{AL}=\mathrm{SV} 3$ was $1.153 \mathrm{SV}$ units, somewhat less than the corresponding groups in Experiment 1, while the mean shift for groups with training $A L=S V 6$ was -.479 , somewhat more than in Experiment 1 . Overall, there was no evidence of a change in mean shift $[F(1,285)=3.72, p>.05]$; that is, increasing training by a factor of four did not reduce $A L$ shift measurably. Apparently, the extent to which increased exposure reduces AL shift depends on the stimulus dimension employed. For example, Hébert, Origlio, and McGuirk (1972) did not observe anchoring with additional training using lifted weights, while Guirintano (cited in Thomas \& Fenner, 1978) reported an anchoring effect with the stimulus dimension of line orientation.

However, with the increased training, there was a substantial differential peak shift (see Table 1, Column 7) of more than $1 / 2$ SV unit in both groups in the directions predicted by Spence's theory. The groups with training $A L=S V 3$ gave a differential shift of $.612 \mathrm{SV}$ units $[F(1,285)=5.15, p<.05]$ and those with training $A L=S V 6$ had a differential shift of $.696 \mathrm{SV}$ units $[\mathrm{F}(1,285)=6.67, \mathrm{p}<.05]$.

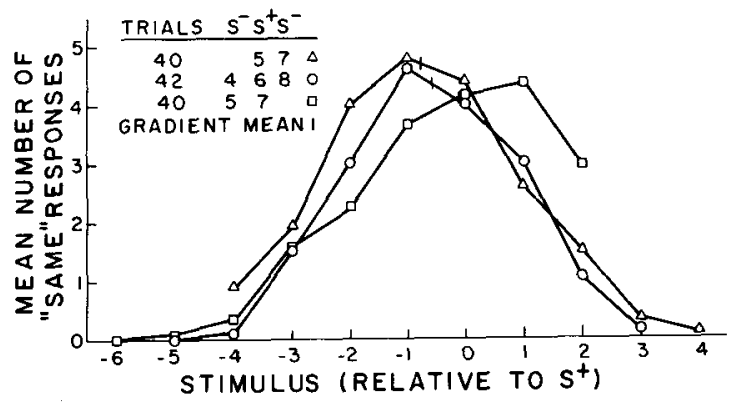

Figure 3. Generalization gradients and gradient means (mean of individual subjects' gradient means; see text for further explanation) for groups with extended discrimination training and training ALs of SV 6. S+ is labeled zero on the abscissa.

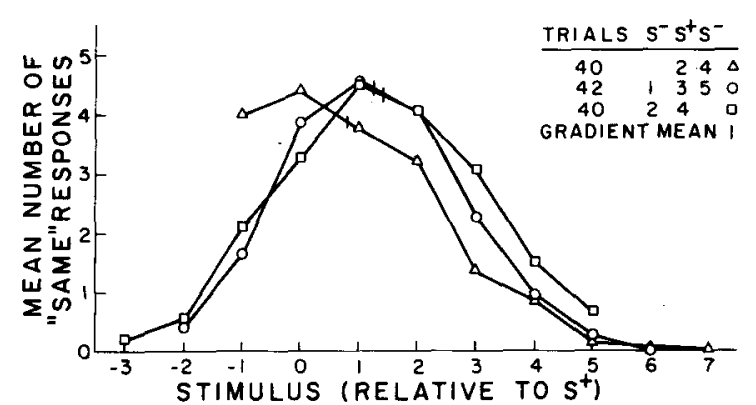

Figure 4. Generalization gradients and gradient means (mean of individual subjects' gradient means; see text for further explanation) for groups with extended discrimination training and training ALs of SV $3 . \mathrm{S}+$ is labeled zero on the abscissa.

This differential shift is shown graphically for the training $\mathrm{AL}=\mathrm{SV} 6$ groups in Figure 3. Here the group with high $\mathrm{S}+(\mathrm{SV} 7)$ shows a mode $1 \mathrm{SV}$ unit above $S+$ while the group with low $S+$ (SV 5) peaks $1 \mathrm{SV}$ unit below $\mathrm{S}+$. This is the typical shift predicted by Spence's theory (Hanson, 1959). Only the downward shift of the group with centered S+ (SV 6) shows uniquely the contribution of $\mathrm{AL}$ to these groups.

The picture is a little different for the groups with training $A L=S V ~ 3$, as shown in Figure 4. The group with the centered S+ (SV 3) shows the upward shift as predicted by $\mathrm{AL}$ theory. The group with high $\mathrm{S}+$ (SV 4) again shows a mode one unit above $\mathrm{S}+$; however, the group with low S + (SV 2) peaks at its $\mathrm{S}+$, failing to show either the negative shift predicted by Spence's theory or the positive shift predicted by $A L$ theory; these shifts are in opposite direction and should cancel, and indeed they do. These overall results constitute strong evidence for the operation of both AL and Spence-type processes.

\section{EXPERIMENT 3}

In Experiment 3, another aspect of Spence's theory received attention. For this theory to predict shift, it is necessary for excitatory and inhibitory gradients to be formed, which requires that different valences be assigned to the stimuli to be discriminated. If discrimination occurs without differential evaluation, no peak shift should be found. In terms of decision theory, when stimuli are to be identified, a set of (presumably symmetrical) criteria are set along the discriminal distribution. Only when feedback is given for the correctness of particular discriminations (e.g., one $\mathrm{S}+$ and one $\mathrm{S}-$ ) would an asymmetrical change in decision criteria be expected. On the other hand, AL theory predicts shift only on the basis of prior experience with the stimuli independent of their valence. In Experiment 3, we investigated this prediction by having subjects rate the training stimuli on a 7-point brightness scale. This required them to discriminate the stimuli without designating one as posi- 
tive and one as negative and without feedback. The amount of training was the same as that of Experiment 2, where both AL shift and Spence-type shift were found. The subjects were then shown the stimulus to be remembered (the same value as the $\mathrm{S}+$ in Experiment 2 for comparable groups) and a generalization gradient was obtained as before.

\section{Method}

Subjects. The subjects were 30 male and 30 female students enrolled in introductory psychology courses at the University of Colorado.

Apparatus. The apparatus was the same as in Experiment 1.

Procedure. Subjects were unsystematically assigned to three groups corresponding to the training $A L=S . V 6$ groups of Experiment 2.

After the subject was seated in front of the stimulus panel, the following instructions were read: "This is an experiment in brightness perception. A light will be presented repeatedly through a small hole in the screen in front of you. Each time it will be presented for five seconds and may have a different brightness. Your first task is to rate the brightnesses of the light. Each time the light is presented, give me a number from one to seven that represents how bright the light seems to you. Let 'one' stand for very dim, 'seven' for very bright, and 'four' for a medium brightness. Remember, each time the light is presented it will stay on for only five seconds. So try to respond while the light is on, using a number from one to seven with numbers less than four representing lights that seem dim, numbers above four representing lights that seem bright, and numbers around four representing lights that seem to have a medium brightness. Any questions?" Only question dealing with the procedure were answered by the experimenter. Following any needed clarifications of instructions, subjects who were to be shown SV 5 or SV 7 as test stimulus were shown 20 series of two stimuli (SV 5 and SV 7) and subjects who were to be shown SV 6 as test stimulus were shown 14 series of three stimuli (SV 4, SV 6, and SV 8).

Next, the following instructions were read to all subjects: "That was the end of the rating task. The next brightness is called the test brightness. Try to remember this brightness because you will have to distinguish it from all the other brightnesses. When you do recognize the test brightness, say 'same.' If a subsequent brightness is different from the test brightness, say 'different.' Remember each time the light is presented it will stay on for only five seconds so try to respond while the light is on. The next light is the test brightness. Keep that brightness in mind and for every light after that say only 'same' or 'different'. Any questions?'" After any further clarification of procedure, the test stimulus was presented and then a generalization gradient was obtained by presenting six series of all nine stimuli, as in Experiments 1 and 2.

\section{Results and Discussion}

In analyzing the data of Experiment 3, mean shift and differential shift were computed as if the subjects had received discrimination training. As can be seen in Table 1, an estimate of AL shift of $-.488 \mathrm{SV}$ units was obtained, which does not differ from that obtained in the analogous groups in Experiments 1 and $2[\mathrm{~F}(1,285)=.388]$. However, the differential shift is in the opposite direction from that predicted by Spence's theory. Thus, we can conclude that a Spence-type shift is not obtained when discrimination occurs without differential evaluation and feedback.

\section{GENERAL DISCUSSION}

It is quite clear that neither Spence's theory (or a reformulation in terms of decision theory) nor $\mathrm{AL}$ theory as they are presently formulated can handle all of the data of this study. As far as we know, only AL theory can account for the major shift in the gradients reported in each experiment, i.e., that which we have labeled "mean shift for triad." This shift is consistent with the claim that as $\mathrm{AL}$ changes from its training to its test value during the course of testing, subjects should continue to respond maximally to the stimulus which bears the same relationship to the current $\mathrm{AL}$ as the $\mathrm{S}+$ did to training $\mathrm{AL}$. On the other hand, the reliable differential shift found in Experiment 2, as well as the failure to find such a shift in Experiment 3 is consistent with Spence's view of the discrimination learning process. It seems clear that two separate processes account for these two shifts, a perceptual (AL) process and a gradient interaction process or decision criterion change.

It may seem odd that we have tested predictions with adult human subjects which were derived from a theory proposed over 40 years ago to account for transposition in lower animals. In human developmental studies, Kendler and Kendler (1959) and Kuenne (1946), working with transposition and with the difference between intra- and extradimensional shifts, respectively, found Spence's (1937) theory to be applicable only to young children. Spence's theory, however, continues to dominate accounts of animal discrimination learning, which is where the bulk of the peak shift literature is found. Furthermore, the most recent published paper in this area, by Galizio and Baron (1979), was represented as a test between Spence-type and AL interpretations. Most importantly, we based our predictions on Spence's theory because it is the only theory explicit enough to generate predictions which could be contrasted with those based on AL theory.

We feel, however, that there are several reasons for suggesting that it is inappropriate to attribute "differential shift" to the interaction of excitatory and inhibitory processes. First, responding in this paradigm is based largely on instructions rather than conditioning. In animal studies of generalization, responding occurs to non-S + values, but at a lower level than to $\mathrm{S}+$, presumably reflecting detection of the difference between the training and test stimuli; in recognition of this, Hull (1943) plotted generalization gradients using an abscissa scaled in JND units. Note, however, that responding in these animal studies occurs to stimuli which are highly discriminably different from the training value. In human studies, subjects are typically given explicit instructions not to respond (or to respond "different") to stimuli which are perceived as different from the training 
stimulus. Therefore, at least in these human studies (assuming that subjects follow instructions), responding to generalized stimuli must be attributed to a failure to perceive a difference. Thus, a perceptual explanation of peak shift in the human studies would seem more appropriate than a learning explanation.

A potential explanation within the framework of the Thomas et al. (1973) theory that the training stimulus is encoded as $\mathrm{S}=\mathrm{AL} \pm \mathrm{X}$ would be to assume that discrimination training increases $X$, i.e., the stimuli involved in a discrimination come to be perceived as farther apart (and therefore farther from AL) than stimuli not actively discriminated. The idea that practice makes stimuli more discriminable is not a new one (cf. Gibson, 1969); however, none of these earlier theories distinguish between discrimination training with or without feedback or the separation of the discriminative stimuli. Nor do they predict that the stimuli will be perceived as having more separation as well as being easier to discriminate. From an $\mathbf{A L}$ point of view, it would not be unreasonable to assume that the size of a unit of X might change, following the same rules as for the AL of the stimulus itself. But since there is no reason to believe that this should be affected by discrimination training, the failure to find a differential peak shift in Experiment 3 is inconsistent with this interpretation.

It seems likely that some modification of Hébert's (1973) model, taking into account asymmetrical decisive criteria produced by discrimination training with feedback, is needed to account for the results of the present experiments and those of Galizio and Baron (1979) and Thomas et al. (1973). Such a modification would suggest that subjects have a range of discriminal processes around $\mathrm{AL} \pm \mathrm{X}$ to which they respond as though the training stimulus has been presented (i.e., "same"). When training is relatively brief and the difference between training $\mathrm{AL}$ and test $\mathrm{AL}$ is relatively large, as it was in Thomas et al. (1973) and in Experiment 1 of this study, one would expect the dominant shift to be due to the change in adaptation level. With sufficient training with a single $\mathrm{S}-$, as in Experiment 2 of this study, a shift due to asymmetry of decision criteria would also be found. With still further training, such as is found in animal studies, where the AL is so strongly anchored by the training stimuli that it is not affected by the test stimuli, one would expect to find a shift principally attributable to changes in decision criteria.

\section{REFERENCE NOTE}

1. White, K.G., \& Thomas, D. R. Postdiscrimination stimulus generalization and adaptation level in humans. Unpublished manuscript, 1979.

\section{REFERENCES}

Blough, D. S. Generalization gradient shape and summation in steady-state test. Journal of the Experimental Analysis of Behavior, 1969, 12, 91-104.

Boneau, C. A., \& Cole, J. L. Decision theory, the pigeon, and the psychological function. Psychological Review, 1967, 74, 123-135.

Capehart, J., Tempone, V. J., \& Hébert, J. A. A theory of stimulus equivalence. Psychological Review, 1969, 76, 405-418.

Galizio, M., \& BARON, A. Human postdiscrimination gradient: The effects of three-stimulus discrimination training. Animal Learning \& Behavior, 1979, 7, 53-56.

GiBson, E. J. Principles of perceptual learning and development. New York: Appleton-Century-Crofts, 1969.

Hanson, H. M. Effects of discrimination training on stimulus generalization. Journal of Experimental Psychology, 1959, 58, 321-334.

HéberT, J. A. Adaptation-level and theory of signal detection: An examination and integration of two judgment models for voluntary stimulus generalization. Acta Psychologica, 1973, 37, 15-29.

Hébert, J. A., Origlio, D. P., \& McGuirk, F. P., III. Training and testing effects in the generalization of a voluntary response. Psychonomic Science, 1972, 26, 209-210.

Helson, H. Adaptation-level as a frame of reference for prediction of psychophysical data. American Journal of Psychology, 1947, 60, 1-29.

Helson, H. Adaptation level theory. New York: Harper \& Row, 1964.

Hull, C. L. Principles of behavior. New York: Appleton-CenturyCrofts, 1943.

JAMES, H. An application of Helson's theory of adaptationlevel to the problem of transposition. Psychological Review, 1953, 46, 345-351.

Kendler, T. S., \& Kendler, H. H. Reversal and non-reversal shifts in kindergarten children. Journal of Experimental Psychology, 1959, 58, 56-60.

KRECHEVSKY, I. A study of the continuity of the problem solving process. Psychological Review, 1938, 45, 107-133.

Kuenne, M. Experimental investigation of the relation of language to transposition behavior in young children. Journal of Experimental Psychology, 1946, 36, 471-490.

Newlin, R. J., Rodgers, J. P., Dickson, J. F., Strub, H., \& Thomas, D. R. The central tendency effect in stimulus generalization: Effects of establishing a "preexperimental" frame of reference. Perception \& Psychophysics, 1978, 24, 161-167.

Parducci, A. Range-frequency compromise in judgment. Psychological Monographs, 1963, 77, No. 2.

Parducci, A. \& Perret, L. S. Category rating scales: Effects of relative spacing and frequency of stimulus values. Journal of Experimental Psychology, 1971, 89, No. 2.

Purtee, R. B. Peak shift: A review. Psychological Bulletin, 1973, 80, 408-421.

Spence, K. W. The differential response in animals to stimuli varying within a single dimension. Psychological Review, 1937, 44, 430-444.

Thомаs, D. R. The effects of drive and discrimination training on stimulus generalization. Journal of Experimental Psychology, $1962,64,24-28$

Thомаs, D. R. The role of adaptation-level in stimulus generalization. In G. Bower (Ed.), The psychology of learning and motivation (Vol. 8). New York: Academic Press, 1974.

Thomas, D. R., \& Fenner, D. P. The central tendency effect in stimulus generalization: The effect of sex of subject. American Journal of Psychology, 1978, 91, 509-522.

Thomas, D. R., \& Jones, C. G. Stimulus generalization as a function of the frame of reference. Journal of Experimental Psychology, 1962, 67, 77-80. 
Thomas, D. R., Svinicki, M. D., \& Vogt, J. Adaptation level as a factor in human discrimination learning and stimulus generalization. Journal of Experimental Psychology, 1973, 97, 210-219.

\section{NOTES}

1. It should be noted that Helson (1964) suggested that the AL might be a function of the geometric mean of a series of stimuli. Parducci (1963) and Parducci and Perrett (1971) have challenged this formulation, suggesting that the range (which determines midpoint) and frequency of stimulus presentation (which determines median), need to be combined in order to properly predict $A L$. In the studies presented here, geometric mean, median, and midpoint vary together. Further, AL was determined empirically by asking separate groups of subjects to rate each series of stimuli used and taking as the estimate of $\mathrm{AL}$ the stimulus rated 4 (on a scale of 1 to 7), determined by a linear regression of stimulus values on ratings. Thus, the differences between Helson's and Parducci's formulations are irrelevant to the present considerations.
2. Using independent gorups in a rating task, $A L$ was found to be consistently somewhat above the geometric mean of each series. (See also, Newlin, Rodgers, Dickson, Strub, \& Thomas, 1978, and Thomas, Svinicki, \& Vogt, 1973.)

3. Since the gradients have more values below the $S+$ value than above, the mean is slightly biased in favor of this result. However, the statement would remain true if subjects' modes or means of symmetrical gradients were used.

4. This study was actually conducted as one large experiment with 15 groups, as indicated in Table 1 . It is reported here as three experiments for ease of exposition. $F$ tests given in the text are planned orthogonal comparisons. For the overall experiment, $F(14,285)=34.47, p<.01$. For the $t$ tests, the combined estimate of variance was used yielding $285 \mathrm{df}$. When sex was included as a factor in the analysis of the experiments, there were no significant sex effects.

(Received for publication December 14, 1978; revision accepted March 14,1979 .) 